\title{
Diseño e implementación de una universidad abierta caso Universidad Central del Ecuador
}

\author{
Design and implementation of a university open case Central \\ University of the Ecuador
}

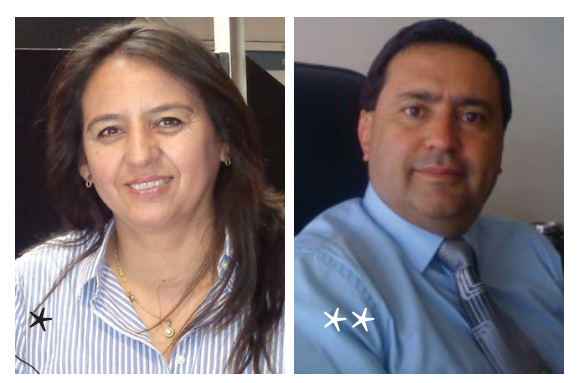

Recibido: 08-oct-2016 Aprobado: 18-dic-2016

ARTÍCULO ORIGINAL

\author{
*Susana Cadena \\ Ing. de Sistemas (ESPE) Docente UCE. Candidata a Ph.D., Universidad de Alicante España. \\ scadena@uce.edu.ec \\ ${ }^{* *}$ Robert Enríquez \\ Ing. en Electrónica y telecomunicaciones (EPN), docente UCE. Candidato a Ph.D., Universidad de \\ Alicante España. \\ renriquez@uce.edu.ec
}

\section{Resumen}

La Universidad del Central del Ecuador se encuentra en un cambio trascendental en su vida institucional con el fin de retomar la categoría A en la evaluación del CEAACES. Esta acreditación tiene un componente importante en el desarrollo de la investigación y conocimiento. Considerando estos aspectos, se ha diseñado una propuesta basada en el uso de la tecnología y que ha sido implementada como una estrategia integral, orientada hacia una universidad abierta que permita sustentar los principios de Transparencia, Participación, Colaboración. El logro de esta estrategia está supeditado a la implementación de tecnología de punta que consolide estos principios en el seno de la comunicad universitaria. Este artículo detalla las etapas a implementarse en una Universidad Abierta, considerando los siguientes momentos: estado del arte, descripción del proyecto, metas logradas, siguientes pasos, y un resumen de las lecciones aprendidas. Se presenta la experiencia de implementación de los proyectos que soportan la estrategia y cómo ellos se han ido diseñando e implementando

Palabras clave: universidad abierta; datos abiertos; calidad de datos

\section{Abstract}

The Central University of Ecuador is undergoing a major change in its institutional life to return to Category A in its evaluation by the CEAACES. This proposal presents an analysis of the following questions: Where do we go? Where are we? How can we get there? The responses to these questions are framed on the principles of an open university based on e-government, open government and open data concepts. The technology takes a vital role in this proposal, where the roadmap is drawn to achieve the goal. The experience of implementing projects and how they have been designed, implemented and improved in a virtuous circle is presented. It includes a general framework of the Integrated Information System, Connectivity, Data Center which are the basic infrastructure that will implement the strategy in the University. Achievements, next steps and lessons learned are also included. 


\section{Importancia del proyecto}

Este proyecto es importante para la Universidad Central del Ecuador por los motivos que se describen a continuación:

- Proporcionará información abierta y disponible, considerando que los datos son de diferentes tipos de fuentes que están conectados a una red, por ejemplo: las cámaras de tráfico, estaciones meteorológicas, dispositivos informáticos conectados a Internet, páginas www y contenido de los servicios de redes sociales (Jaakkola, Mäkinen, \& Eteläaho, 2014).

- La explotación de datos en formatos abiertos, generado por un proceso electrónico, que significa la traducción de datos desde una aplicación informática a un conocimiento útil que puede proporcionar varios tipos de apoyo en la toma de decisiones (Milić, Veljković, \& Stoimenov, 2012).

- Permitirá alinear la inversión en infraestructura tecnológica a la academia y a la investigación, orientada en el proceso de acreditación de las instituciones de educación superior instaurado a partir del año 2008.

- Servirá para definir una estrategia que permita la rendición de cuentas a la comunidad, la participación y la colaboración que son los principios del Gobierno Abierto.

- Permitirá el desarrollo del conocimiento a partir de la implementación de datos abiertos, ya que los avances tecnológicos y las comunicaciones en todo el mundo, en tiempo real, son una gran promesa para la transformación de la eficiencia y eficacia de los servicios públicos a través de la facilidad de publicación y acceso a la información pública (dos Santos Brito, da Silva Costa, Garcia, \& de Lemos Meira, 2014).

\section{Estado de arte}

El Gobierno Abierto propone la transparencia, y la forma práctica es, mediante la apertura de datos donde deberían asentarse las bases para que las universidades empiecen a abrir los datos en forma coherente, desarrollando políticas de transparencia y de acceso abierto al conocimiento, tanto para docentes como para investigadores (Teruel Doñate, 2014). (Naser \& Concha, 2013)

Este concepto toma impulso a partir de las siguientes declaraciones:

Declaración del G8 en Lough Erne (18 de junio de 2013), que es un conciso decálogo de políticas sobre la responsabilidad de los gobiernos. Incluye un punto sobre apertura y reutilización de información.

Los tres principios fundamentales de este movimiento, recogidos en el celebrado "Memorando de Obama" (2009), son:

- Transparencia: oferta de información clara y actualizada, accesible y reutilizable.
- Participación: intervención de la ciudadanía en todas las actividades del gobierno.

- Colaboración: entre instituciones y convocatoria a los ciudadanos a compartir lo que saben y a generar soluciones en las áreas donde tienen conocimientos.

En la legislación ecuatoriana:

En la Guía de Política Pública en Datos Abiertos (Ecuador, 2014) se describe la relación entre Gobierno Abierto, Datos Abiertos, y Política Nacional de Gobierno Electrónico y el uso de buenas prácticas en Datos Abiertos para transparencia y participación ciudadana.

Ley del sistema nacional de registro de datos públicos, en donde se establece la forma que el Estado ecuatoriano administrará los datos.

Ley Orgánica de Transparencia y Acceso a la Información Pública (Ecuador, 2014)

"Una Universidad Abierta es aquella que implementa iniciativas de gobierno abierto respaldando el acceso a la información generada por la institución, facilitando la participación por medio de canales o herramientas TI y ayudando al intercambio de información entre las administraciones, fomentando así la transparencia en beneficio de todos" (Aparicio Juan Manuela, Fuster Andrés, Garrigós Irene, Maciá Francisco, Mazón José Norberto, Vaquer Llorenc, 2015).

La Universidad Abierta es aquella que favorece el acceso abierto a la información, escucha a la comunidad universitaria, tomando decisiones según sus necesidades y prioridades, y genera espacios de colaboración para desarrollar servicios con todos los actores de la comunidad universitaria (Teruel Doñate, 2014).

La apertura en la universidad, por tanto, se hace necesaria y se manifestará además en otros ámbitos complementarios, como el acceso abierto a los resultados científicos, la educación en formato abierto, o mediante procesos participativos/abiertos en sus acciones de gobierno. "Estos requerimientos son un camino que ya ha sido emprendido en EEUU y Europa, Io que se propone, en un espíritu de reutilización, que tiene los siguientes principios que indican que los datos deben ser:

- Abiertos por defecto

- Oportunos y exhaustivos

- Accesibles y utilizables

- Comparables e interoperables

- Para mejorar la gobernanza y la participación ciudadana

- Para el desarrollo incluyente y la innovación" (Open Data Charter, 2015). 
Publicar datos en formatos abiertos requiere lo siguiente:

- Usar esquemas y vocabularios consensuados y utilizar metadatos abiertos.

- Inventario en un catálogo de datos estructurado.

- Datos accesibles desde direcciones web persistentes y amigables.

- Exponer un conjunto mínimo de datos relativos al nivel de competencias del organismo y su estrategia de exposición de datos.

- Compromiso de servicio, actualización y calidad del dato, manteniendo un canal eficiente de comunicación para una reutilización de datos.

- Monitorear y evaluar el uso y servicio mediante métricas.

- Datos bajo condiciones de uso no restrictivas y comunes.

- Socializar y educar en el uso de datos.

- Recopilar aplicaciones, herramientas y manuales para motivar y facilitar la reutilización (CRUE, 2011).

\section{Descripción del proyecto}

\section{Objetivo general}

Implementar una Universidad Abierta considerando los principios de gobierno abierto.

\section{Objetivos específicos}

- Diseñar los elementos de una Universidad Abierta

- Diseñar e implementar la infraestructura tecnológica

- Implementar los componentes de colaboración y participación.

\section{Antecedentes}

Considerando las metas que tiene la institución en los siguientes años para diseñar el proyecto, se analizó los elementos de un gobierno abierto que son: Participación, Colaboración y Trasparencia, además se consideró la misión y visión de la institución que son:

\section{- Visión}

La Universidad Central del Ecuador continuará en el liderazgo de la educación superior, de la producción de ciencia, tecnología, cultura y arte y en la formación profesional con profunda responsabilidad social.

\section{- Misión}

La Universidad Central del Ecuador forma profesionales críticos de nivel superior, comprometidos con la verdad, justicia, equidad, solidaridad, valores éticos y morales; ge- nera ciencia, conocimiento, tecnología, cultura y arte; y, crea espacios para el análisis y solución de los problemas nacionales.

Además de estos elementos, que son el marco de referencia para el proyecto, se tomó en consideración que los proyectos de inversión tecnológica vayan más allá de la automatización de servicios, que apuntalen el desarrollo estratégico de la Institución, que su meta fundamental es la formación académica de grado y posgrado; por tanto, este proyecto se orienta hacia la creación de una Universidad Abierta, considerando las siguientes premisas:

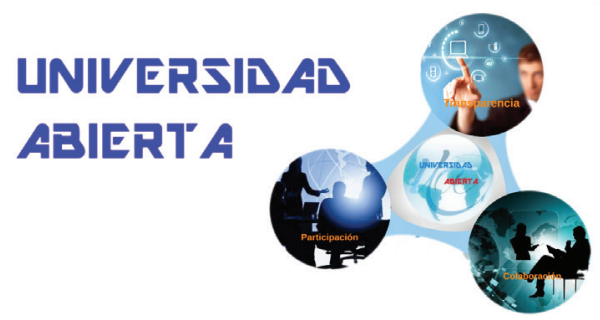

Figura 1. Estructura del Proyecto Universidad Abierta, caso Universidad Central

Estos aspectos nos ayudarán a garantizar que se cree en la universidad un ecosistema que permita desarrollar la colaboración, participación y transparencia, de acuerdo a lo siguiente:

\section{Transparencia}

Para lograr este objetivo se trabajaron los siguientes elementos que están ya están disponibles:

- Nuevo sitio web institucional. Se generó un nuevo portal con un espacio para transparencia en donde se refleja la información año por año.

- Biblioteca. Se implementó un repositorio digital para publicar los datos de las tesis de pregrado y posgrado, además de los resultados de las investigaciones. Dbspace. uce.edu.ec

- Sistema Académico. Se realizó un proceso de calidad de datos con el propósito de publicar la información de estudiantes, para este proceso se ejecutó convenios con la Dinardap, y se trabajó con cada una de las facultades, al momento los datos ya son públicos en Universidad en Cifras. www.uce.edu.ec

- Revista Digital. Se creó un espacio para publicar las revistas digitales de la institución en el sitio revistadigital. uce.edu.ec

- Se realizó una alianza con Microsoft y se lanzó el servicio de correo electrónico con varias herramientas que permitan la publicación de información personal e institucional.

\section{Participación}

Para lograr este objetivo se trabajaron los siguientes elementos que ya se encuentran disponibles: 
Se creó un portal de encuestas universitarias en donde se puede generar información de carácter público y privado. encuestas.uce.edu.ec

- Redes Sociales. La Dirección de Comunicación y Cultura está a cargo del manejo de las redes sociales oficiales de la Universidad Central del Ecuador, en una segunda etapa se analizará las tendencias de estas redes.

- Redes Sociales apoyadas en el correo electrónico. Se puso al servicio de la comunidad la herramienta Yamer, Formularios, Grupos, Sitios, con el fin de que la comunidad universitaria pueda generar espacios de participación. Mail.uce.edu.ec, con esto se logró una integración de las facultades.

- $\quad$ Clubes. Se dio un espacio en la página web www.uce. edu.ec, a los clubes de andinismo y ciencias, rugby, que ya están presentes como un espacio de participación.

- Comité de Ética. La Universidad creó esta unidad con el propósito de generar la participación académica, de investigación y Revista Digital. Se creó un espacio para publicar las revistas digitales de la institución en el sitio revistadigital.uce.edu.ec

\section{Colaboración}

Para lograr este elemento se trabajaron los siguientes instrumentos que están en proceso de construcción:

- Portal de Datos Abiertos. Es un espacio donde se publicarán los datos en formatos abiertos, con el propósito de que la comunidad reutilice esta información.

- Proyecto de Conectividad y Smart City. Estos son subproyectos que se encuentran en ejecución.

- Laboratorio de Innovación. Es un espacio en donde le permite a la Universidad colaborar con otros sectores e intrega a la comunidad. Universitaria.

- Sistema Integral de Información. Es un elemento en donde se generará la información de todos los actores. Se analizará la calidad de la información antes de la publicación en el portal.

Sistema integral de información

- Sistema de Registro Funcionarios es un repositorio de los docentes, sus trabajos de investigación y sus logros.

- Correo electrónico institucional para las 50 mil personas que constituyen la comunidad universitaria, se trabajó con Microsoft 365 en la nube, y es con el active directory el single sign on de la universidad para autentificarse en todas las aplicaciones. Se empezó con 470 hábiles diarias hasta Ilegar ahora a las 10.600 hábiles diarias.
- Implementación de acceso único para las bases científicas, los docentes y estudiantes pueden acceder desde sus casas a las bases. Se empezó con una tasa de acceso de 3.500 y en la actualidad tenemos más de 150.000

- Implementación de un sistema de Business Intelligence para reportes gerenciales.

\section{Red inalámbrica y campus}

La estrategia de Universidad Abierta requiere tener una universidad digital que permita en forma práctica enlazar todas las iniciativas que se presenten en los tres principios, para esto es fundamental una infraestructura sólida, siendo la conectividad un pilar entre toda la comunidad universitaria.

¿Cómo llegar a esa conectividad a través de la implementación de una red tanto alámbrica como inalámbrica? Se necesita que todas las Facultades y la Administración Central se encuentren conectadas a un mismo sistema único donde se pueda establecer las políticas necesarias para garantizar la transparencia, colaboración y participación de la comunidad universitaria.

Para ello se desarrolló un diseño total de conectividad de la Universidad que tiene varios componentes:

- Integración de las Facultades y la Administración Central a través de un campus conectado con fibra óptica a $10 \mathrm{G}$.

- Implementación de una red inalámbrica para todo el campus con alrededor de 900 access point, para garantizar el acceso a Internet y a la intranet.

- Diseños de conectividad interna de cada Facultad y dependencia administrativa.

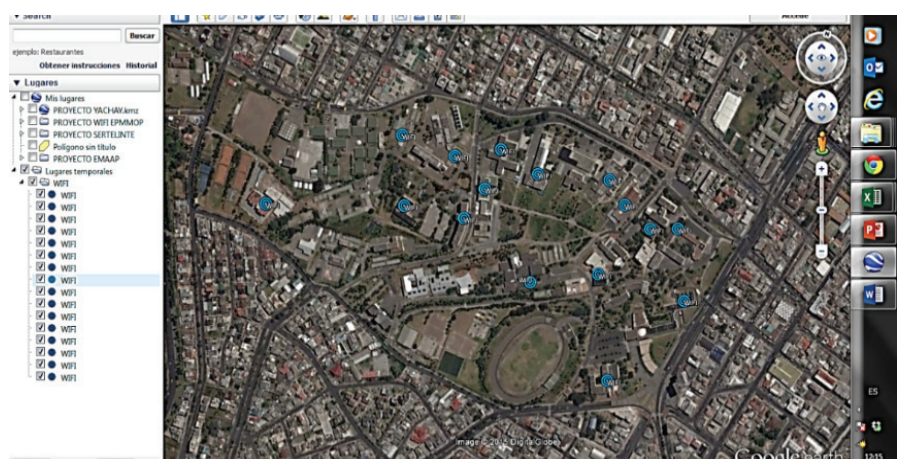

Figura 2. Ubicación del Campus Universitario

El objetivo fue crear una red inalámbrica para la Universidad Central del Ecuador que sea centralizada, que permita la conexión de puntos de acceso, puntos de distribución y Core para la comunicación de sus facultades y sedes.

\section{Desarrollo}

- Definición de metodología de trabajo para la creación de 
un ecosistema de innovación.

- Creación de dispositivos que permitan obtener bases de datos en tiempo real de variables a controlar y monitorizar.

- Concursos (hackathon) para creación de aplicaciones que utilicen los datos, con el fin de beneficiar a la universidad y a la comunidad educativa.

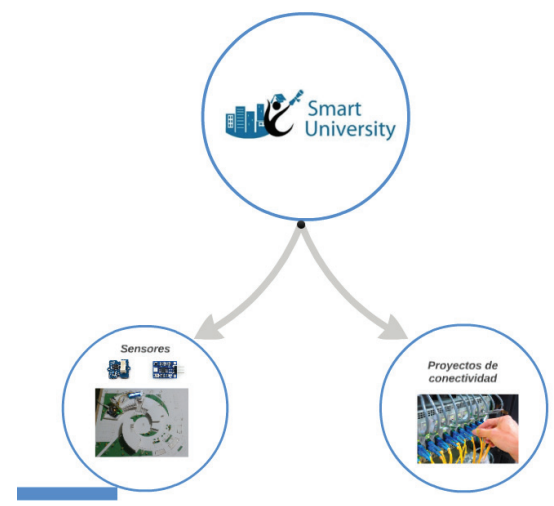

Figura 8. Proyectos Smart University

\section{Logros}

- Primera Hackathon de innovación para aplicaciones que permitan llevar a la universidad a una Universidad Inteligente. Las aplicaciones que se presentaron fueron:

- Medición de radiaciones UV.

- Sistema de control de inventarios con RFID para el Laboratorio de innovación UCE LAB.

- Sistema de registro de asistencia con sensores de presión implementados en sillas.

- Sistemas de control de ingreso de alumnos con RFID y huella.

- Sistema de apertura de puertas con RFDI, aplicación móvil.

- Sistema de basureros inteligentes con sensores de llenado y sensores de acercamiento.

- Bastón con sonido para ayuda de personas no videntes.

- Sistema de riego automático con implementación de sensores de humedad.

- Sistema de parqueaderos con sensores de ultrasonido.

- Registro de asistencia con analítica de video implementada en cámara IP.

- Implementación de tres proyectos con data sets.

- Proyecto de medición de rayos UV, aplicación móvil y creación de data sets en tiempo real.

- Proyecto de control de inventarios con RFID.
- Proyecto de basureros inteligentes.

- Metodología para la creación de proyectos, mapa de datos y reutilización de los mismos.

\section{Siguientes pasos}

- Para los siguientes años la Universidad Central del Ecuador deberá ejecutar las siguientes acciones:

- Poner en funcionamiento el portal de datos abiertos.

- Implementar el ecosistema de innovación y socializar la plataforma de seguimiento de proyectos de innovación.

- Implementar el Sistema Integral de información.

En la siguiente gráfica se presenta el ecosistema plateado para el Laboratorio de innovación UCE LAB.

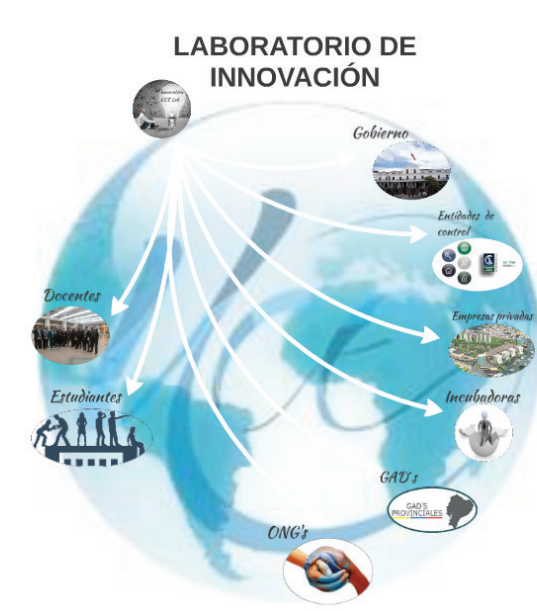

Figura 9. Laboratorio de Innovación

\section{Lecciones aprendidas}

\section{Lección 1. Enfocarse en las necesidades del negocio}

- Problema. De las implementaciones de tecnología realizadas en la Universidad, se observa que las que no han tenido éxito son aquellas que no están enfocadas en el negocio y partieron de una necesidad tecnológica, sin considerar la participación de los usuarios de propietarios del proceso de negocio, ocasionando que los proyectos no tengan el apoyo requerido, ya que se lo mira como gasto en tecnología y no como una inversión del negocio.

- Propuesta. Para tener un mayor éxito de los proyectos de tecnología se trabajó con los usuarios los procesos que se encontraban a cargo, levantando el (As Is) y mejorando el mismo con el equipo, los reglamentos internos, las leyes, y la estrategia de la Universidad, logrando los procesos To $\mathrm{Be}$, los mismos que luego se automatizaron.

\section{Lección 2. Sostenibilidad de los proyectos del negocio}

- Problema. Una vez que se genera un proyecto para satisfacer una necesidad del negocio no se piensa en la 
sostenibilidad del mismo en el tiempo.

- Propuesta. En los proyectos desarrollados en la Universidad se estableció un análisis de costo/beneficio con indicadores financieros y no financieros, que admitan la sostenibilidad en el tiempo incluyendo servicios que permitan desarrollar las áreas gobernantes de la académica en nuestro caso son: Docencia, Investigación, Vinculación y Gestión.

\section{Lección 3. Implementación de tecnología de alto impacto en corto tiempo}

- Problema. Los proyectos tecnológicos como infraestructura y desarrollo de aplicaciones necesitan alta inversión, estudios de factibilidad, diseño e implementación, lo que hace que sean de largo aliento, ocasionando pérdida de credibilidad en los responsables del negocio, que en el caso de la Universidad tienen un período de administración de cinco años.

- Propuesta. En los proyectos desarrollados en la Universidad se definió proyectos de alto impacto, con bajo costo que incrementaron la credibilidad, mientras se desarrollaba los proyectos bases, por ejemplo: Página web institucional con estándares internacionales de accesibilidad, usabilidad rescatando de la identidad centralina.

\section{Lección 4. Usabilidad de las aplicaciones}

- Problema. Las diferentes aplicaciones se realizan en base al criterio técnico del informático a cargo de proyecto, por tanto, el momento de su implementación no logran el impacto esperado en los usuarios, en su uso y optimización de servicios del negocio.

- Propuesta. Para el diseño de las aplicaciones se orientó al servicio, teniendo como prioridad la usabilidad de las aplicaciones, para lo cual, se incorporó en el equipo un diseñador de aplicaciones WEB y un administrador de empresas experto en levantamiento de procesos y servicios; con este profesional se diseñó los prototipos después de diseñar los servicios. Luego que fue aprobado el prototipo por el usuario final se inició la etapa de programación; cuando esta etapa concluyó, antes de poner a producción, se estableció que el usuario final ponga el aprobado de la misma, garantizando la usabilidad. Al usuario le interesa el servicio y no la tecnología que está atrás.

\section{Lección 5. Involucrar a la máxima autoridad}

- Problema. Debido al costo de los proyectos tecnológicos y lo abstracto de la tecnología no son entendidos por las autoridades y por tanto no hay el apoyo.

- Propuesta. Transformamos los proyectos tecnológicos a un lenguaje propio de las autoridades, demostrando objetividad de los mismos como una estrategia institucional a largo plazo, logrando de esta manera el apoyo de las autoridades, y con su liderazgo la realización de los proyectos.

\section{Lección 6. Alta capacidad técnica del equipo de tecnología}

- Problema. Los equipos de tecnología no poseen habilidades de liderazgo y conocimientos de última tecnología.

- Propuesta. Realizamos una selección de técnicos con experiencia y se les capacitó en manejo de proyectos, desarrollando habilidades de liderazgo, lo cual impactó en el éxito de los proyectos.

\section{Referencias bibliográficas}

Aparicio Juan Manuela, Fuster Andrés, Garrigós Irene, Maciá Francisco, Mazón José Norberto, Vaquer Llorenc, Z. J. J. (2015). Ecosistema de Datos Abiertos de la Universidad de Alicante. (U. de Alicante, Ed.). Alicante.

CRUE. (2011). ¿Hacia una Universidad Abierta?, 1-35.

Dos Santos Brito, K., da Silva Costa, M. A., Garcia, V. C., \& de Lemos Meira, S. R. (2014). Brazilian government open data. Proceedings of the 15th Annual International Conference on Digital Government Research - Dg.0 '14, 11-16. http://doi. org/10.1145/2612733.2612770

Ecuador, I. G. M. (2014). República del Ecuador. Quito: Secretaría Naciona de la Administración Pública - Ecuador.

Jaakkola, H., Mäkinen, T., \& Eteläaho, A. (2014). Open Data: Opportunities and Challenges. Proceedings of the 15th International Conference on Computer Systems and Technologies - CompSysTech '14, 25-39. http://doi. org/10.1145/2659532.2659594

Mili, P., Veljkovi, N., \& Stoimenov, L. (2012). Framework for open data mining in e-government. Proceedings of the Fifth Balkan Conference in Informatics on - BCl '12, 255. http:// doi.org/10.1145/2371316.2371369

Naser, A., \& Concha, G. (2013). El gobierno electronico en la gestion publica. Journal of Chemical Information and Modeling, 53, 1689-1699. http://doi.org/10.1017/ CB09781107415324.004

Open Data Charter. (2015). International Open Data Charter, (September), 8. Retrieved from http://opendatacharter.net

Teruel Doñate, E. (2014). Hacia una Universidad Abierta, 1(Hacia Una Universidad Abierta), 1-35. 